\title{
Encapsulation of Moringa oleifera Extract in Ca-Alginate Chocolate Beads: Physical and Antioxidant Properties
}

\author{
Olga Kaltsa $(\mathbb{D}$, Aggeliki Alibade $\mathbb{D}$, Eleni Bozinou $(\mathbb{D}$, Dimitris P. Makris $\mathbb{D}$, \\ and Stavros I. Lalas \\ Department of Food Science \& Nutrition, University of Thessaly, Terma N. Temponera Street GR-43100, Karditsa, Greece \\ Correspondence should be addressed to Stavros I. Lalas; slalas@uth.gr
}

Received 27 February 2021; Revised 6 May 2021; Accepted 28 May 2021; Published 4 June 2021

Academic Editor: Walid Elfalleh

Copyright ( $\odot 2021$ Olga Kaltsa et al. This is an open access article distributed under the Creative Commons Attribution License, which permits unrestricted use, distribution, and reproduction in any medium, provided the original work is properly cited.

\begin{abstract}
The aim of the present study was to evaluate the physical and antioxidant properties of chocolate alginate beads containing Moringa oleifera leaf extract (MLE) produced with ecofriendly solvent extraction technology (Deep Eutectic Solvents). The concentration of MLE incorporated was $0,2,4$, and $6 \% \mathrm{w} / \mathrm{w}$, and hardening time for ionotropic gelation with $\mathrm{CaCl}_{2}$ solution was 2, 8, or 20 min. Freshly prepared beads were evaluated for their geometric (area, perimeter, ferret diameter, circularity, roundness), color (CIE $\mathrm{L}^{*}$, $\mathrm{a}^{*}$,

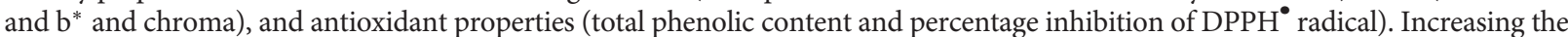
MLE concentration resulted in beads smaller in size and more spherical, whereas hardening time only affected their circularity. MLE concentration had also a profound effect on color and antioxidant properties of the beads. As the concentration of MLE increased, the beads appeared lighter and their chroma increased. The radical scavenging activity was ameliorated by the MLE concentration increase for samples hardened for 8 and $20 \mathrm{~min}$, whereas it was unaffected for those at $2 \mathrm{~min}$. The hardening time on the contrary did not affect the inhibition of $\mathrm{DPPH}^{\bullet}$ values, regardless of the amount of extract added.
\end{abstract}

\section{Introduction}

Within the last decade, there is an increasing interest in functional, health-promoting foods from all three parts involved: science, food industry, and consumers. As most processes during food preparation contribute to significant degradation of vital compounds (phytochemicals, antioxidants, vitamins) and probiotics, a significant number of techniques have been developed, focusing on the encapsulation of these sensitive constituents. In addition, the encapsulation of bioactive ingredients offers the advantage of targeted delivery and controlled release in the human gastrointestinal tract, which increase their bioefficiency [1]. Only to begin with, nano-/microemulsification, multiple emulsion formation, liposome entrapment, spray drying, lyophilization, ionotropic gelation, and coacervation represent the most widely known [2]. Emerging electrohydrodynamic techniques like electrospinning and electrospraying are also promising tools for fabrication of fibrous or sphere-shaped vehicles $[2,3]$.
Moringa oleifera Lam. (drumstick tree) is a plant native to northern India, largely appreciated for its uses in traditional medicine. Although every part of the tree is useful, there is growing interest for its leaf extract because of the high concentrations in certain antioxidant compounds such as vitamin $\mathrm{E}$, ascorbic acids, $\beta$-carotene, quercetin, phenolic acids, and flavonoids [4-6]. A plethora of in vivo and in vitro studies provide significant evidence on the prophylactic effect against common diseases, including Alzheimer's, atherosclerosis, diabetes, and neoplasms [7]. With respect to its antioxidant properties, an appreciable number of studies have been conducted within the last few years [8-10]. Moringa oleifera extract has also been successfully encapsulated using different techniques in various systems, such as electrospun nanofibers [11], microemulsions [12], phytosomes [13], and spray-dried capsules [14].

The ionotropic gelation of alginates in the presence of $\mathrm{Ca}^{2+}$ or $\mathrm{Mg}^{2+}$ by the cross-linking mechanism is a very useful affordable encapsulation technique for food and nonfood applications that offers, among others, advantages such as 
biodegradability, biocompatibility, and implementation simplicity [15]. In addition, the size, shape, and controlled release of encapsulated materials can be easily tailored to meet the needs of specific applications, i.e., drugs or nutraceuticals. This can be achieved by altering the chemical structure of the encapsulant, procedure variables, and matrix composition [16-18]. As a consequence, the encapsulation of widely used herbal extracts rich in polyphenols (olive leaf, pomegranate, beetroot, and cocoa) in Ca-alginate hydrogels has been the subject of an appreciable number of studies [19-22]. Wine by-product extract [23] and medicinal extracts of Plectranthus species have also been successfully encapsulated in similar systems $[24,25]$.

The limited reports that exist on encapsulation of Moringa leaf extract (MLE) in alginate beads focus on the removal of heavy metals $[26,27]$ or their bactericidal activity [28] and lack vital information related to their physical, antioxidant properties, and possible application in food.

Therefore, the main scope of this work was to encapsulate MLE, prepared with the use of deep eutectic solvents (DES) in chocolate-flavored alginate beads, via ionic gelation to enhance their antioxidant capacity. The effect of selected parameters (curing time and extract concentration) on the physical and antioxidant properties of the alginate beads is discussed.

\section{Material and Methods}

2.1. Materials. Folin-Ciocalteu reagent, 1,1-diphenyl-2picryl-hydrazyl $\left(\mathrm{DPPH}^{\bullet}\right)$, glacial acetic acid, $\mathrm{CaCl}_{2}$, and $\mathrm{CaCO}_{3}$ were purchased from Sigma-Aldrich (St. Louis, MO, USA). Gallic acid hydrate and Folin-Ciocalteu was from Panreac (Barcelona, Spain). Cocoa butter and whey protein concentrate $(80 \%$ in protein) were kind donations from Cocoowa (Thessaloniki, Greece) and Tyrokomiki Karditsas S.A. (Karditsa, Greece), respectively. Cocoa powder was obtained from a local store. Alginate powder was donated by Kenfood S.A. (Keratsini, Greece), MLE was prepared under the optimum conditions previously reported by Zam et al., [20] using DES. In the same study, a detailed analysis of the chemical properties of the MLE (total polyphenol and flavonoid content, antiradical activity, ferric reducing power, and polyphenolic profile by LC-MS and HPLC) is provided.

\subsection{Methods}

2.2.1. Preparation of Chocolate Beads Containing MLE. The chocolate used to formulate the MLE-containing alginate beads was prepared according to the main methodology for chocolate manufacture that involves four different phases: dry mixing, grinding, conching, and molding [29]. It contained $36 \% \mathrm{w} / \mathrm{w}$ cocoa butter, $32 \% \mathrm{w} / \mathrm{w}$ sugar, $12 \% \mathrm{w} / \mathrm{w}$ cocoa powder, and $20 \% \mathrm{w} / \mathrm{w}$ whey protein concentrate.

Finally, alginate chocolate beads were prepared as follows: sodium alginate powder $(2 \mathrm{~g})$ was dispersed in double distilled water $(60 \mathrm{~g})$ containing $20 \mathrm{~g}$ chocolate, $0.5 \mathrm{~g}$ potassium sorbate, and $0.5 \mathrm{~g}$ potassium benzoate. The mix was vigorously stirred for $45 \mathrm{~min}$ at $500 \mathrm{rpm} / 50^{\circ} \mathrm{C}$. The alginate dispersion was left to cool at ambient temperature, MLE was added at $0,2,4$, and $6 \% \mathrm{w} / \mathrm{w}$ concentration, and water was topped up to $100 \mathrm{~g}$ for each formulation. The spherification of the final alginate dispersion was carried out with plastic pipettes, and the dripping procedure in $\mathrm{CaCl}_{2}$ solution $(2 \%$ w/w) lasted $3 \mathrm{~min}$. The distance between the pipette nozzle and the surface of the $\mathrm{CaCl}_{2}$ solution was $2 \mathrm{~cm}$. The beads were maintained in the $\mathrm{CaCl}_{2}$ solution to gel for 2,8 , or $20 \mathrm{~min}$ and afterward filtered and washed with double distilled water to remove excess calcium and stop hardening. Curing time intervals were selected with preliminary testing in order to correspond to different types of texture: samples cured for $2 \mathrm{~min}$ were very soft and easily broken upon slight pressure with the tongue, samples of $8 \mathrm{~min}$ represent more cohesive beads, and samples of $20 \mathrm{~min}$ were hard spheres that required extensive chewing to disintegrate. All samples were prepared and analyzed at least in triplicate.

2.2.2. Chocolate Beads Particle Size. The particle size of the beads was estimated by means of image analysis. Photos of the beads' samples were captured with a digital camera coupled with a computer. The free license image analysis software ImageJ v. 1.46r (https://imagej.nih.gov/ij/index. html) was used to calculate the geometric properties of the beads: area $\left(\mathrm{mm}^{2}\right)$, ferret diameter $(\mathrm{mm})$, perimeter $(\mathrm{mm})$, roundness $(-)$, and circularity $(-)$. For each measurement, approximately 200 beads were measured per batch.

2.2.3. Color Measurement. Color properties of chocolate beads were estimated by a color and appearance measurement system (Lovibond CAM-System 500; Great Britain). The system was calibrated using the GretagMacbeth mini color checker model P/N:50111 to obtain $\mathrm{L}^{*}, \mathrm{a}^{*}$, and $\mathrm{b}^{*} \mathrm{CIE}$ values. The chroma (C) was also calculated according to equation (1) as follows [30]:

$$
\operatorname{Chroma}(C)=\left(\left(a^{*}\right)^{2}+\left(b^{*}\right)^{2}\right)^{0.5} \text {. }
$$

2.2.4. Total Phenolic Content (TPC). The TPC of alginate beads was determined by the Folin-Ciocalteu method with modifications $[4,31]$. Briefly, $0.5 \mathrm{~g}$ of beads was mixed with $10 \mathrm{ml}$ of $1 \% \mathrm{w} / \mathrm{v}$ acetic acid solution, homogenized using ULTRA-TURRAX (T25, IKA) at 10,000 rpm for $30 \mathrm{sec}$. The mixture was finally centrifuged $4,000 \mathrm{rpm} / 10 \mathrm{~min}$ ), and supernatants were collected for further analysis. An aliquot of $100 \mu \mathrm{l}$ of the supernatant was mixed with $100 \mu \mathrm{l}$ of FolinCiocalteu reagent in a $1.5 \mathrm{ml}$ Eppendorf tube and allowed to react for $2 \mathrm{~min}$. After that, $800 \mu \mathrm{l}$ of $\mathrm{Na}_{2} \mathrm{CO}_{3}$ solution $(5 \%$ $\mathrm{w} / \mathrm{v}$ ) was added, and the tubes were incubated in a water bath for $20 \mathrm{~min}$ at $40^{\circ} \mathrm{C}$. The absorbance of the samples was recorded at $740 \mathrm{~nm}$ using appropriate blanks containing formic acid solution. Total phenolic content concentration (TPC) was estimated by using a gallic acid calibration curve $(10-80 \mathrm{mg} / \mathrm{ml})$. The results were expressed as gallic acid equivalents (mg GAE/g of beads). 


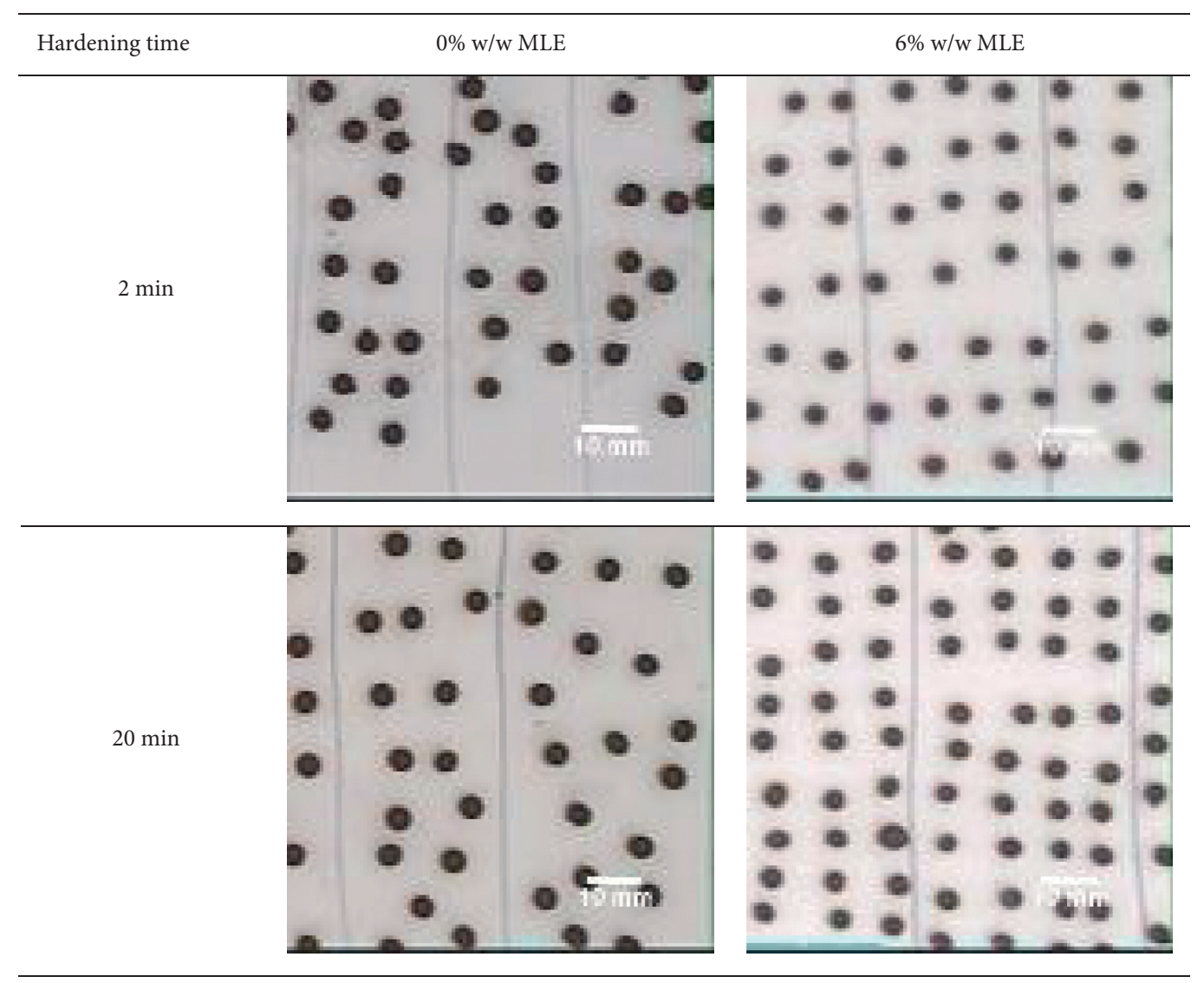

Figure 1: Appearance of chocolate alginate beads as affected by MLE concentration and hardening time.

2.2.5. Radical Scavenging Activity Determination. The antioxidant activity of chocolate beads was evaluated by scavenging of $\mathrm{DPPH}^{\bullet}$. A $25 \mu \mathrm{l}$ aliquot of the beads' supernatant prepared as described previously for TPC analysis was mixed with $925 \mu \mathrm{l}$ freshly prepared $\mathrm{DPPH}^{\bullet}$ solution $(80 \mathrm{mM})$. The mixture was incubated for $30 \mathrm{~min}$ at room temperature $\left(25^{\circ} \mathrm{C}\right)$, and the absorbance was measured at $515 \mathrm{~nm}$.

The radical scavenging activity of the beads was finally estimated according to [32], and the results are expressed as percentage of inhibition (I\%) against blank according to equation (2):

$$
(I \%)=100 \times \frac{\left(A_{0}-A_{s}\right)}{A_{0}},
$$

where $A_{0}$ and $A_{\mathrm{s}}$ are the absorbances at $515 \mathrm{~nm}$ of the blank and sample, respectively.

2.2.6. Sensory Evaluation. Sensory evaluation was performed by a panel of 15 experienced members ( 8 female, 7 male). The $9-$ point hedonic scale $(1=$ extremely dislike, $5=$ nor like nor dislike, and $9=$ extremely like) was used to evaluate selected samples of chocolate beads (control and samples with $6 \% \mathrm{w} / \mathrm{w}$ MLE) in terms of color, taste, aroma, texture, and general acceptance [33].

2.2.7. Statistical Analysis. All physicochemical analyses were performed in triplicate. The statistical analysis was performed with StatGraphics Centurion XV software by means of analysis of variance (ANOVA). Fisher's least significant difference (LSD) test was used to discriminate among sample means at 95\% level of confidence $(p<0.05)$.

\section{Results and Discussion}

3.1. Chocolate Bead Size. Manufacturing parameters (nozzle diameter, distance of the nozzle from the gelling solution, temperature, polymer solution feed rate, cross-linking agent concentration) and alginate properties (polymer concentration, molecular weight) can be modified to alter or optimize the geometric properties of the beads [34, 35].

In Figures 1 and 2(a)-2(d), the appearance and geometric properties of control and MLE chocolate beads are demonstrated. For presentation reasons in Figure 1, only concentration ( 0 and $6 \% \mathrm{w} / \mathrm{w}$ MLE) and hardening time extremes (2 and $20 \mathrm{~min}$ ) are depicted. The Feret diameter, defined as the maximum caliper of an object, varied between 4.30 and $5.02 \mathrm{~mm}$ (Figure 2(a)). A gradual decrease of the beads' diameter was observed by increasing the MLE concentration from 0 to $6 \% \mathrm{w} / \mathrm{w}$. The diameter of control beads ranged between $\sim 4.8$ and $5.0 \mathrm{~mm}$, whereas those at maximum MLE concentration were around $4.3 \mathrm{~mm}$ wide. The same trend was observed for area and perimeter (Figure 2(b) and $2(\mathrm{c}))$ that decreased by almost 22 and $12 \%$, respectively, when MLE concentration increased from 0 to $6 \% \mathrm{w} / \mathrm{w}$. Similar findings have been referred in other studies on 


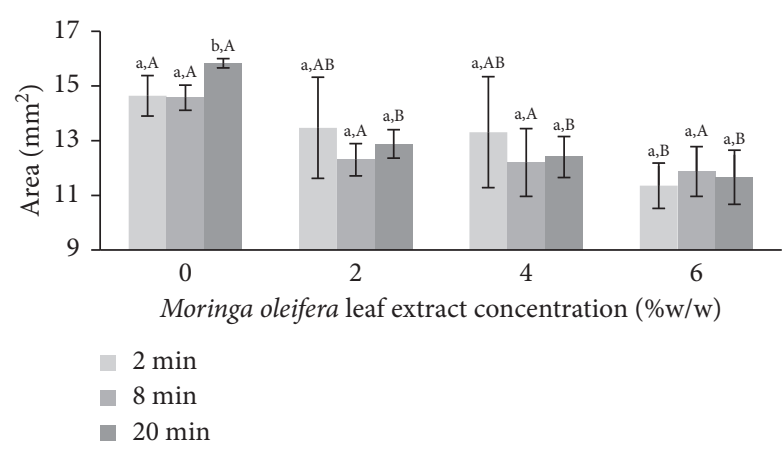

(a)

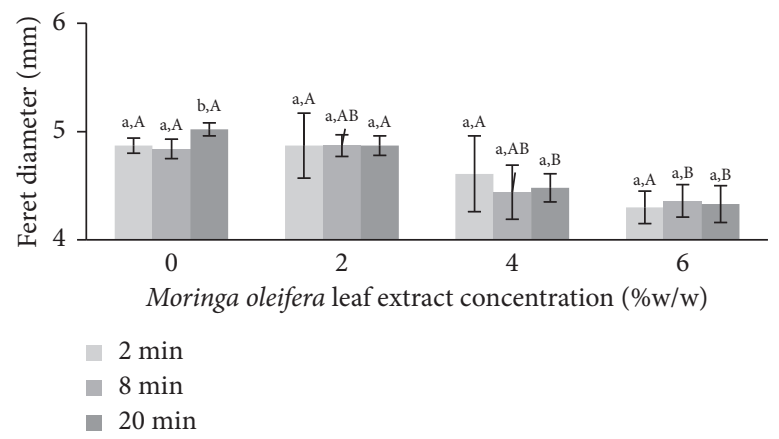

(c)

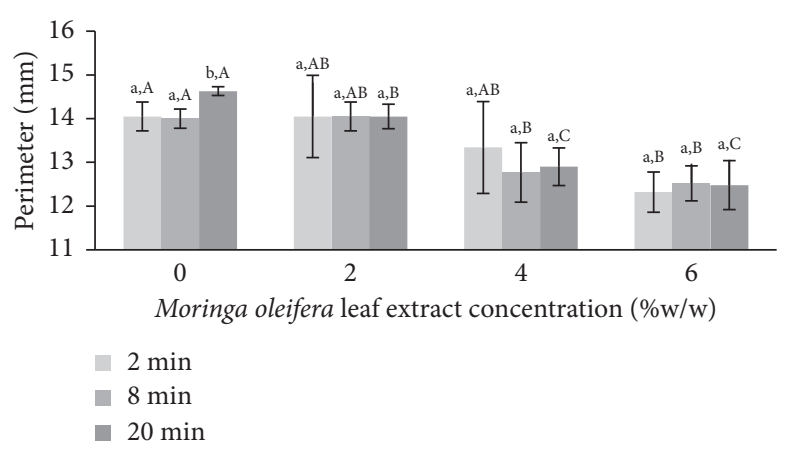

(b)

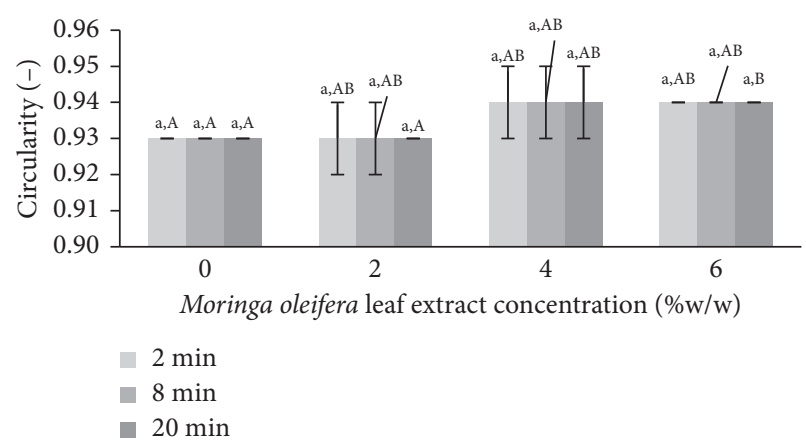

(d)

FIgURe 2: Geometric properties of chocolate alginate beads as affected by MLE concentration $(0,2,4$, and $6 \% \mathrm{w} / \mathrm{w})$ and hardening time $(2,8$, and $20 \mathrm{~min}$ ). Different small letters indicate significant differences among samples of the same concentration, while different capital letters indicate significant differences among samples of the same hardening time at $p<0.05$.

alginate beads with added extracts or phenolic compounds. Kannat et al. [36] reported that the size of alginate beads was reduced from 2.42 to $2.05 \mathrm{~mm}$ upon the addition of onion scale extract from 0 up to $6 \% \mathrm{w} / \mathrm{w}$. Smaller bead size was also observed for beads with incorporated caffeine [37]. It has been also documented that several phenolic compounds have the ability to lower the surface tension of solutions, which may explain the observed decrease in size [38].

However, increasing the curing time from 2 to $20 \mathrm{~min}$ did not result in beads smaller in size $(p<0.05)$. The opposite phenomenon has been referred in other studies. As shown elsewhere, the time allowed for gelation in calcium chloride bath had a major effect on bead size, but the influence on shape is less intense [39]. Long curing time resulted in smaller beads due to extensive cross-linking that increased the rigidity of the bead matrix. In the same study, it is also recommended that the total dripping time is limited to 2-3 min and curing time between $15-20 \mathrm{~min}$ to avoid excessive shrinkage and increased polydispersity within the sample. Smaller and more spherical beads were obtained with prolonged curing time up to $30 \mathrm{~min}$ [35].

Circularity values equal to 1 indicate geometries of a perfect circle, while those approaching 0 correspond to elongated shapes. A slight increase in circularity values from 0.93 to 0.94 was detected by increasing the MLE concentration, although significant differences compared with control samples were observed only when $6 \% \mathrm{w} / \mathrm{w}$ of extract was added. Hence, less elongated beads were produced at maximum MLE concentration.
It could be therefore concluded that the glycerol contained in the MLE is the main factor governing the size and the rest geometrical properties of the alginate beads, rather than the curing time.

3.2. Color Properties. Color is considered a key property for food products as it can affect other important sensory characteristics related to consumer acceptance and purchasing intention $[40,41]$.

Figure 3(a)-3(d) shows the influence of MLE concentration and hardening time on the color properties $\left(\mathrm{L}^{*}, \mathrm{a}^{*}\right.$, $\left.\mathrm{b}^{*}\right)$ and chroma of alginate beads. $\mathrm{L}^{*}, \mathrm{a}^{*}$, and $\mathrm{b}^{*}$ values of all samples prepared ranged between 30.7 and 39.3, 8.1 and 13.2 , and 6.6 and 11.3, respectively, resembling the color of chocolate bars [42]. Peak values for lightness were observed at $4 \% \mathrm{w} / \mathrm{w}$ MLE concentration $\left(\mathrm{L}^{*}=38.4-39.3\right)$, whereas slightly lower values were observed at $6 \% \mathrm{w} / \mathrm{w}$. An increase of redness and yellowness (positive $\mathrm{a}^{*}$ and $\mathrm{b}^{*}$ values) was also observed by increasing MLE concentration of the alginate solution up to $4 \% \mathrm{w} / \mathrm{w}$, but it remained practically unaffected at the maximum MLE concentration. The same trend was also observed for chroma, as it is a property generated by $\mathrm{a}^{*}$ and $\mathrm{b}^{*}$ values. This is due to the pigments present in the MLE that impart a yellowish appearance to it. Interestingly, the trend observed for the lightness of the beads as affected by MLE content correlated with that observed for TPC (Figure 4). More specifically, as TPC initially decreased, an increase in $\mathrm{L}^{*}$ values was observed and the 


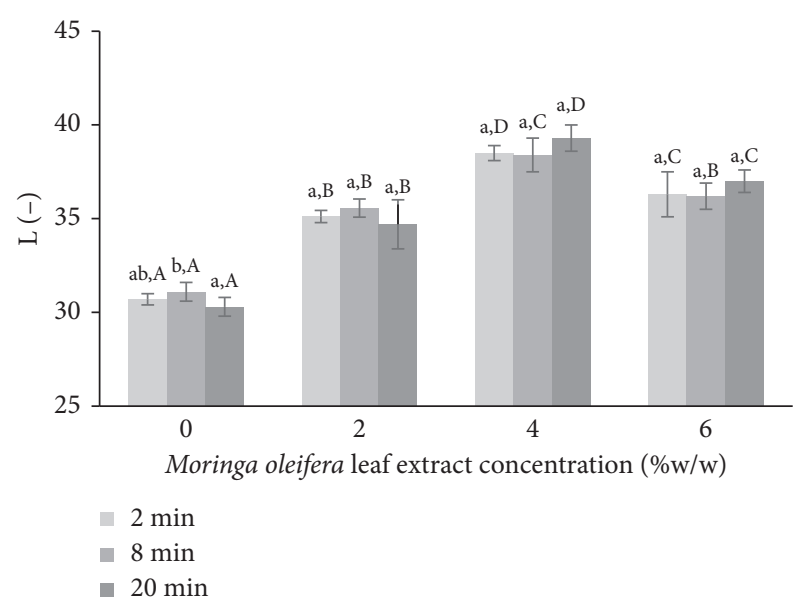

(a)

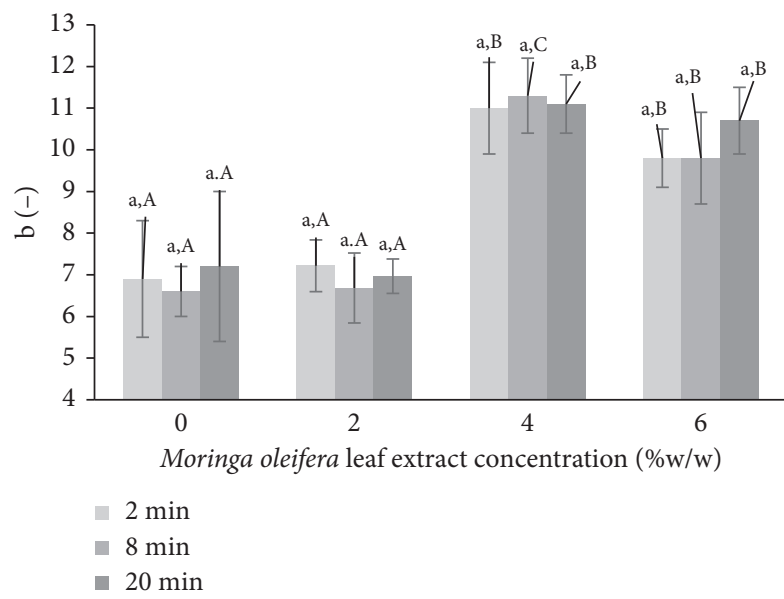

(c)

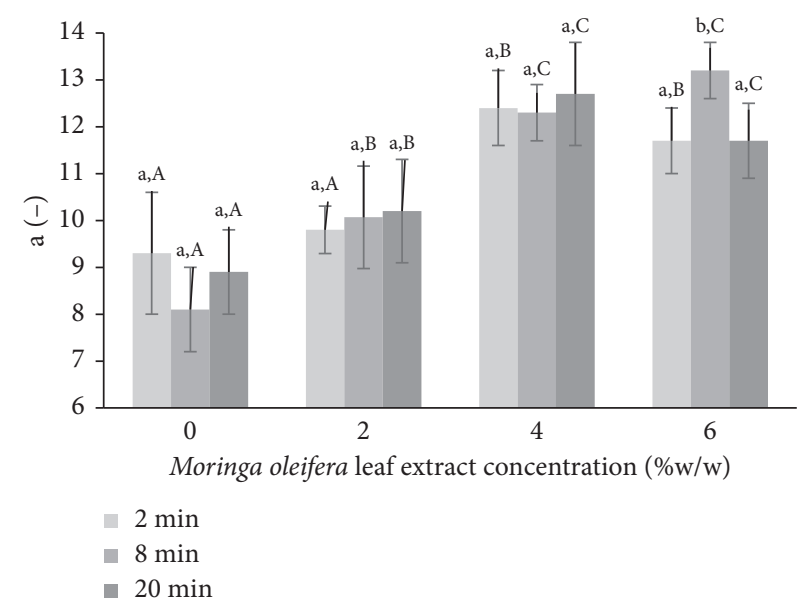

(b)

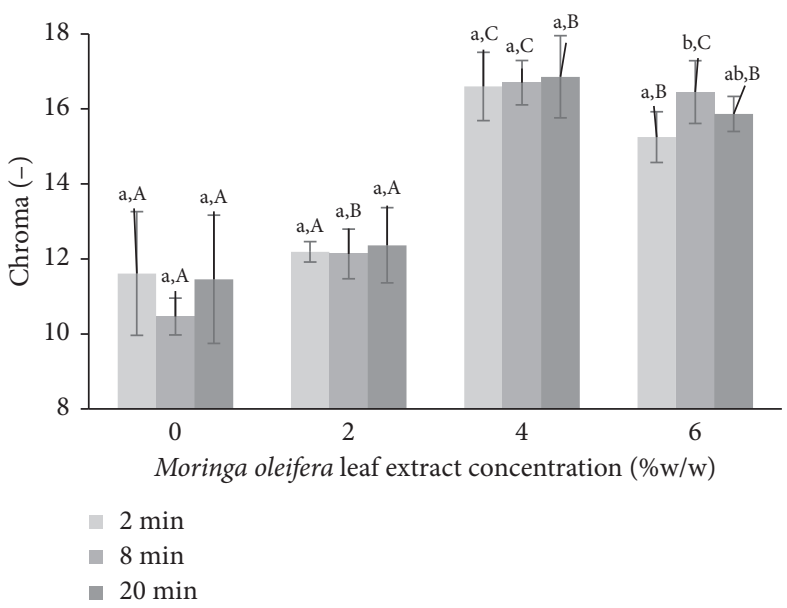

(d)

FIGURE 3: CIE L*, $\mathrm{a}^{*}$, and $\mathrm{b}^{*}$ and chroma color properties of chocolate alginate beads as affected by MLE concentration $(0,2,4$, and $6 \% \mathrm{w} / \mathrm{w})$ and hardening time $(2,8$, and $20 \mathrm{~min})$. Different small letters indicate significant differences among samples of the same concentration, while different capital letters indicate significant differences among samples of the same hardening time at $p<0.05$.

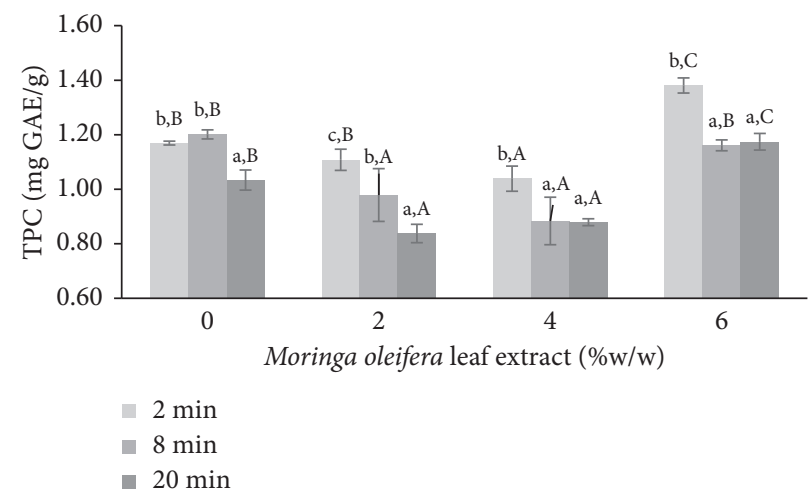

Figure 4: Total phenolic content (TPC) of chocolate alginate beads as affected by MLE concentration (0, 2, 4, and 6\% w/w) and hardening time (2, 8, and $20 \mathrm{~min})$. Different small letters indicate significant differences among samples of the same concentration, while different capital letters indicate significant differences among samples of the same hardening time at $p<0.05$. 


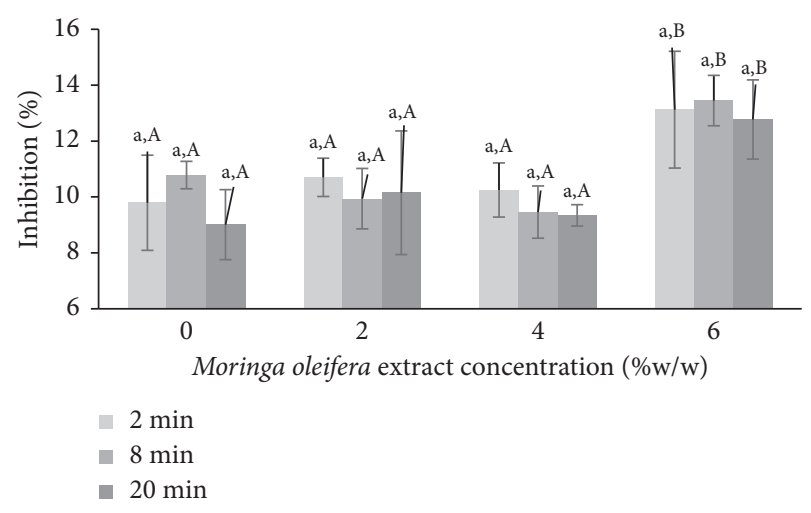

FIGURE 5: Radical scavenging capacity (I\%) of chocolate alginate beads as affected by MLE concentration $(0,2,4$, and $6 \% \mathrm{w} / \mathrm{w})$ and hardening time (2, 8, and $20 \mathrm{~min})$. Different small letters indicate significant differences among samples of the same concentration, while different capital letters indicate significant differences among samples of the same hardening time at $p<0.05$.

subsequent increase of phenolic compounds ( $6 \%$ w/w MLE) corresponded to decreased $\mathrm{L}^{*}$. This was also observed in other studies [43]. However, the TPC decrease observed upon increasing of hardening time did not alter the $\mathrm{L}^{*}$ values as expected. Therefore, a more detailed study is needed to fully elucidate the effect of MLE on lightness, as other types of compounds such as melanoidins may contribute to the occurring color changes. Melanoidins are high-molecularweight dark brown derivatives formed during the roasting of cocoa beans due to polyphenol degradation through the Maillard reaction [44].

For the vast majority of samples, the time allowed for cross-linking in the ionic solution did not significantly affect the color properties of the samples $(p>0.05)$.

3.3. Total Phenolic Content (TPC). Process parameters that may affect polyphenol loading of alginate beads, apart from extract concentration, include sodium alginate concentration, calcium chloride concentration, and exposure time [20].

In Figure 4, the effect of extract concentration and hardening time on TPC content of alginate beads is depicted. In total, the phenolic content of the samples varied between 0.88 and $1.38 \mathrm{mg}$ GAE/g of beads. Similar contents have been reported in other studies involving the encapsulation of herbal extract in alginate beads [45]. Both parameters studied (MLE concentration and hardening time) had a profound effect on TPC values. Although an increase in phenolic content would be expected by increasing the extract concentration in the alginate solution, this was not observed in our case. A gradual decrease of TPC was observed upon increase of the extract added up to $4 \% \mathrm{w} / \mathrm{w}$, after which it was finally increased. This trend was observed in all cases regardless of the hardening time used. The above phenomenon could be induced by the presence of high amounts of glycerol in the beads, which is the main component of DES used for the preparation of the Moringa extract. Glycerol is a known plasticizer with low molecular weight.
Glycerol concentration above a critical value, which depends on the molecular weight of alginates, may induce chemical interactions that modify the mechanical, morphological, and barrier properties of alginate polymer films [46, 47]. The small size of glycerol facilitates its penetration between alginate hydrogen bonds, resulting in reduced intermolecular forces that increase the mobility of the polymer chains. As a consequence, less compact polymer matrices are formed with increased elasticity [48]. As shown by Ion [49], the increased firmness in alginate-chitosan complexes was caused by enhanced intermolecular interactions of carboxylate groups found in alginates and the amine groups of chitosan. This favored the entrapment of polyphenols during the ionotropic gelation, as evidenced by higher encapsulation efficiency rates compared with control beads that did not contain chitosan.

Hardening time also had a considerable effect on the TPC of the beads. As seen in Figure 4, significantly lower TPCs were observed after 20 min of immersion in the calcium solution in all cases of samples compared with beads exposed for $2 \mathrm{~min}$. This 10 -fold time increase resulted in TPC values decreased by $\sim 16 \%$ on average. Hence, the minimum and maximum phenolic content were observed in samples containing $2 \% \mathrm{w} / \mathrm{w}$ MLE treated for $20 \mathrm{~min}$ and samples with $6 \% \mathrm{w} / \mathrm{w}$ extract treated in $\mathrm{Ca}^{2+}$ solution for 2 min.

As previously reported, calcium chloride exposure time may decrease the loading efficiency of several compounds. Indeed, prolonged exposure may cause a shift of $\mathrm{Ca}^{2+}$ bound within the alginate matrix or excessive release of polyphenols [20].

3.4. Antioxidant Activity. The $\mathrm{DPPH}^{\bullet}$ radical scavenging activity of MLE chocolate beads prepared by varying extract concentration and $\mathrm{CaCl}_{2}$ immersion time is shown in Figure 5. As can be seen, the effect of MLE concentration on the antioxidant activity of the beads is related to the curing time used for sample preparation. For beads allowed to harden for $2 \mathrm{~min}$, percentage inhibition (I\%) values slightly increased from $\sim 9.8$ to $13.1 \%$ when increasing MLE concentration, although no significant differences were found among samples $(p>0.05)$. On the contrary, samples treated for 8 and 20 min presented significantly enhanced antioxidant activity when $6 \% \mathrm{w} / \mathrm{w}$ of MLE was added compared with control samples $(p<0.05)$.

Our results also show that the antioxidant activity of the beads was unaffected by different time intervals used for the hardening of the beads for samples with the same extract concentration $(p>0.05)$.

Interestingly, DPPH inhibition values did not correlate with corresponding phenolic content shown previously in Figure 4. More specifically, despite the reduction of TPC observed as hardening time increased from 2 to $20 \mathrm{~min}$, $\mathrm{DPPH}^{\bullet}$ inhibition percentages were similar among samples with the same MLE concentration. In addition, although samples prepared at 2 min had higher TPC, still they were equally potent with the rest of the samples in terms of radical scavenging $(p>0.05)$. 
TABLE 1: Sensory evaluation scores of chocolate alginate beads (control and 6\% w/w MLE) prepared with different hardening times.

\begin{tabular}{lccccc}
\hline & Color & Taste & Aroma & Texture & General acceptance \\
\hline Control & & & & & \\
$2 \mathrm{~min}$ & $7.07 \mathrm{a} \pm 0.88$ & $6.07 \mathrm{a} \pm 0.80$ & $6.80 \mathrm{a} \pm 0.86$ & $6.53 \mathrm{ab} \pm 0.64$ & $6.80 \mathrm{a} \pm 0.56$ \\
$8 \mathrm{~min}$ & $7.13 \mathrm{a} \pm 0.52$ & $6.20 \mathrm{a} \pm 0.77$ & $6.53 \mathrm{a} \pm 0.99$ & $7.40 \mathrm{c} \pm 0.51$ & $7.00 \mathrm{a} \pm 0.65$ \\
$20 \mathrm{~min}$ & $7.07 \mathrm{a} \pm 0.70$ & $6.27 \mathrm{a} \pm 0.70$ & $6.73 \mathrm{a} \pm 0.46$ & $6.20 \mathrm{a} \pm 0.77$ & $6.87 \mathrm{a} \pm 0.64$ \\
\hline $6 \% \mathrm{MLE}$ & & & & & \\
$2 \mathrm{~min}$ & $7.40 \mathrm{a} \pm 0.51$ & $6.27 \mathrm{a} \pm 0.80$ & $6.47 \mathrm{a} \pm 0.74$ & $6.87 \mathrm{~b} \pm 0.35$ & $7.07 \mathrm{a} \pm 0.46$ \\
$8 \mathrm{~min}$ & $7.00 \mathrm{a} \pm 0.65$ & $5.80 \mathrm{a} \pm 0.86$ & $6.60 \mathrm{a} \pm 0.99$ & $7.67 \mathrm{c} \pm 0.49$ & $7.20 \mathrm{a} \pm 0.41$ \\
$20 \mathrm{~min}$ & $6.73 \mathrm{a} \pm 0.59$ & $6.00 \mathrm{a} \pm 0.85$ & $6.33 \mathrm{a} \pm 0.82$ & $6.53 \mathrm{ab} \pm 0.52$ & $7.20 \mathrm{a} \pm 0.56$ \\
\hline
\end{tabular}

Within the same column, values followed by different letters are significantly different $(p<0.05)$.

A possible explanation would be that the loss of polyphenols caused by prolonged hardening of the beads that wash off from the beads' matrix was not extensive enough to affect their radical scavenging capacity drastically.

On the other hand, as previously referred, melanoidins that result from polyphenol degradation are considered the predominant contributors of the antioxidant activity of cocoa beans [44]. The researchers of this study found that even though TPC of roasted cocoa beans was significantly lower than that of the raw material, the antioxidant activity was the same for both samples. Hence, the reduction in TPC may have not altered the antioxidant potential of the alginate beads detrimentally.

3.5. Sensory Evaluation. Control samples and samples containing $6 \% \mathrm{w} / \mathrm{w}$ MLE were selected for sensory evaluation as the later presented the highest TPC and DPPH values. In Table 1, the scores of different sensory attributes are displayed. In general, all samples were well accepted by panelists, as values for general acceptance, color, taste aroma, and texture ranged between 5.8 and 7.6. The addition of MLE did not impart any unfavorable attributes to the samples as evidenced by similar sensory scores. No significant differences were detected among samples prepared with different curing times in terms of color, taste, aroma, and general acceptance $(p<0.05)$. Findings for color are in line with those demonstrated in Figure 3, showing that since curing time did not alter the color properties of the beads, the panelists showed the same acceptance. On the other hand, curing time extremes (2 and $20 \mathrm{~min}$ ) negatively affected the acceptance of texture as slightly lower values were obtained for these samples. This was observed for both types of samples, control and samples with added MLE. It could be therefore concluded that chocolate beads containing 6\% w/w MLE cured for $8 \mathrm{~min}$ represent the sample with well-accepted sensory attributes and most pleasant texture.

\section{Conclusions}

Moringa oleifera leaf extract (MLE) prepared by a "green" extraction technology, rich in phenolic ingredients, was encapsulated in chocolate-flavored alginate beads. The appearance of the beads including geometric and color properties was affected by the amount of extract incorporated. The higher the amount of MLE added, the higher the redness, yellowness, and chroma of the beads, which also became less elliptical in shape. The amount of MLE added as well as the hardening time was also critical in terms of the phenolic compounds found in the final product. As a result, a minimum TPC concentration of $0.84 \mathrm{mg} \mathrm{GAE} /$ $\mathrm{g}$ beads was established for beads prepared with $2 \% \mathrm{w} / \mathrm{w}$ MLE allowed $20 \mathrm{~min}$ in $\mathrm{CaCl}_{2}$ to harden. However, the radical scavenging activity was only concentration-related and a minimum $6 \% \mathrm{w} / \mathrm{w}$ of MLE is required to enhance its inhibitory effect. The beads prepared with maximum MLE concentration cured for $8 \mathrm{~min}$ had the most favorable texture, so they can be added in food dessert products to enhance their antioxidant potential.

\section{Data Availability}

The data used to support the findings of this study are included within the article.

\section{Conflicts of Interest}

The authors declare no conflicts of interest.

\section{Acknowledgments}

This research has been cofinanced by the European Union and Greek national funds through the Operational Program Competitiveness, Entrepreneurship and Innovation, under the call RESEARCH - CREATE - INNOVATE (project code: T1EDK-05677).

\section{References}

[1] A. Rashidinejad and S. M. Jafari, Handbook of Food Nanotechnology, S. M. Jafari, Ed., Academic Press, Cambridge, MA, USA, pp. 279-344, 2020.

[2] V. Nedovic, A. Kalusevic, V. Manojlovic, S. Levic, and B. Bugarski, "An overview of encapsulation technologies for food applications," Procedia Food Science, vol. 1, pp. 18061815, 2011.

[3] S. Castro Coelho, B. Nogueiro Estevinho, and F. Rocha, "Encapsulation in food industry with emerging electrohydrodynamic techniques: electrospinning and electrospraying-a review," Food Chemistry, vol. 339, Article ID 127850, 2021

[4] A. Lakka, S. Grigorakis, O. Kaltsa et al., "The effect of ultrasonication pretreatment on the production of 
polyphenol-enriched extracts from Moringa oleifera L. (Drumstick tree) using a novel bio-based deep eutectic solvent," Applied Sciences, vol. 10, no. 1, p. 220, 2020.

[5] S. Lalas and J. Tsaknis, "Characterization of Moringa oleifera seed oil variety "periyakulam 1"," Journal of Food Composition and Analysis, vol. 15, no. 1, pp. 65-77, 2002.

[6] A. K. Pandey, R. D. Pandey, P. K. Tripathi, P. P. Gupta, and J. Haider, "Moringa oleifera Lam. (Sahijan) - a plant with a plethora of diverse therapeutic benefits," An Updated Retrospection. Medicinal and Aromatic Plants, vol. 1, pp. 1-8, 2011.

[7] S. Gupta, R. Jain, S. Kachhwaha, and S. L. Kothari, "Nutritional and medicinal applications of Moringa oleifera Lam.review of current status and future possibilities," Journal of Herbal Medicine, vol. 11, pp. 1-11, 2018.

[8] M. Lin, J. Zhang, and X. Chen, "Bioactive flavonoids in Moringa oleifera and their health-promoting properties," Journal of Functional Foods, vol. 47, pp. 469-479, 2018.

[9] M. M. Soliman, A. Aldhahrani, A. Alkhedaide, M. A. Nassan, F. Althobaiti, and W. A. Mohamed, "The ameliorative impacts of Moringa oleifera leaf extract against oxidative stress and methotrexate-induced hepato-renal dysfunction," Biomedicine \& Pharmacotherapy, vol. 128, Article ID 110259, 2020.

[10] F. Wang, S. Long, J. Zhang et al., "Antioxidant activities and anti-proliferative effects of Moringa oleifera L. extracts with head and neck cancer," Food Bioscience, vol. 37, Article ID 100691, 2020.

[11] N. Hani, M. H. Azarian, A. E. Torkamani, and W. A. Kamil Mahmood, "Characterisation of gelatin nanoparticles encapsulated with Moringa oleiferabio active extract," International Journal of Food Science \& Technology, vol. 51, no. 11, pp. 2327-2337, 2016.

[12] G. Batra, O. Gortzi, S. Lalas, A. Galidi, A. Alibade, and G. Nanos, "Enhanced antioxidant activity of Capsicum annuum L. and Moringa oleifera L. extracts after encapsulation in microemulsions," ChemEngineering, vol. 1, no. 2, p. 15, 2017.

[13] A.-W. Lim, P.-Y. Ng, N. Chieng, and S.-F. Ng, "Moringa oleifera leaf extract-loaded phytophospholipid complex for potential application as wound dressing," Journal of Drug Delivery Science and Technology, vol. 54, Article ID 101329, 2019.

[14] P. Robert, T. Gorena, N. Romero, E. Sepulveda, J. Chavez, and C. Saenz, "Encapsulation of polyphenols and anthocyanins from pomegranate (Punica granatum) by spray drying," International Journal of Food Science \& Technology, vol. 45, no. 7, pp. 1386-1394, 2010.

[15] S. Pedroso-Santana and N. Fleitas-Salazar, "Ionotropic gelation method in the synthesis of nanoparticles/microparticles for biomedical purposes," Polymer International, vol. 69, no. 5, pp. 443-447, 2020.

[16] I. Donati, K. I. Draget, M. Borgogna, and S. Paoletti, “Tailormade alginate bearing galactose moieties on mannuronic residues: selective modification achieved by a chemoenzymatic strategy," Biomacromolecules, vol. 6, no. 1, pp. 88-98, 2005.

[17] A. Dodero, L. Pianella, S. Vicini, M. Alloisio, M. Ottonelli, and M. Castellano, "Alginate-based hydrogels prepared via ionic gelation: an experimental design approach to predict the crosslinking degree," European Polymer Journal, vol. 118, pp. 586-594, 2019.

[18] G. A. Islan and G. R. Castro, "Tailoring of alginate-gelatin microspheres properties for oral ciprofloxacin-controlled release against Pseudomonas aeruginosa," Drug Delivery, vol. 21, no. 8, pp. 615-626, 2014.
[19] F. Flamminii, C. D. Di Mattia, M. Nardella et al., "Structuring alginate beads with different biopolymers for the development of functional ingredients loaded with olive leaves phenolic extract," Food Hydrocolloids, vol. 108, Article ID 105849, 2020.

[20] W. Zam, G. Bashour, W. Abdelwahed, and W. Khayata, "Alginate-pomegranate peels' polyphenols beads: effects of formulation parameters on loading efficiency," Brazilian Journal of Pharmaceutical Sciences, vol. 50, no. 4, pp. 741-748, 2014.

[21] B. Lupo, A. Maestro, J. M. Gutiérrez, and C. González, "Characterization of alginate beads with encapsulated cocoa extract to prepare functional food: comparison of two gelation mechanisms," Food Hydrocolloids, vol. 49, pp. 25-34, 2015.

[22] T. R. Aguirre-Calvo, S. Molino, M. Perullini, J. Á. RufiánHenares, and P. R. Santagapita, "Effects of in vitro digestionfermentation over global antioxidant response and short chain fatty acid production of beet waste extracts in $\mathrm{Ca}$ (ii)alginate beads," Food \& Function, vol. 11, no. 12, pp. 10645-10654, 2020.

[23] A. Moschona and M. Liakopoulou-Kyriakides, "Encapsulation of biological active phenolic compounds extracted from wine wastes in alginate-chitosan microbeads," Journal of Microencapsulation, vol. 35, no. 3, pp. 229-240, 2018.

[24] P. Rijo, P. L. Falé, M. L. Serralheiro, M. F. Simões, A. Gomes, and C. Reis, "Optimization of medicinal plant extraction methods and their encapsulation through extrusion technology," Measurement, vol. 58, pp. 249-255, 2014.

[25] A. Belščak-Cvitanović, R. Stojanović, V. Manojlović et al., "Encapsulation of polyphenolic antioxidants from medicinal plant extracts in alginate-chitosan system enhanced with ascorbic acid by electrostatic extrusion," Food Research International, vol. 44, no. 4, pp. 1094-1101, 2011.

[26] M. Franco, J. Beltran-Heredia, and J. E. S. Paterniani, "Use of alginate-Moringa oleifera beads on $\mathrm{Cu}$ (II) and Cd (II) adsorption from aquatic systems," International Journal of Chemical Engineering and Applications, vol. 4, no. 6, pp. 373-376, 2013.

[27] R. Kannan, S. Lakshmi, N. Aparna, S. Prabhakar, and W. R. Thilagaraj, "Eco-friendly treatment of textile dye from aqueous solution using encapsulated biosorbent matrix beads: kinetics and breakthrough analysis," International Journal of Industrial Chemistry, vol. 7, no. 3, pp. 265-275, 2016.

[28] S. E.-H. Asmaa, A. F. Ehab, and G. H. Ahmed, "A study of the comparative antimicrobial activity of Moringa oleifera extracts encapsulated within ALg nanoparticles," Nanoscience \& Nanotechnology-Asia, vol. 10, pp. 1-9, 2020.

[29] T. J. Gutiérrez, "State-of-the-art chocolate manufacture: a review," Comprehensive Reviews in Food Science and Food Safety, vol. 16, no. 6, pp. 1313-1344, 2017.

[30] A. Patras, "Stability and colour evaluation of red cabbage waste hydroethanolic extract in presence of different food additives or ingredients," Food Chemistry, vol. 275, pp. 539-548, 2019.

[31] F.-H. Tsai, Y. Kitamura, and M. Kokawa, "Effect of gum Arabic-modified alginate on physicochemical properties, release kinetics, and storage stability of liquid-core hydrogel beads," Carbohydrate Polymers, vol. 174, pp. 1069-1077, 2017.

[32] A. Lakka, S. Grigorakis, I. Karageorgou et al., "Saffron processing wastes as a bioresource of high-value added compounds: development of a green extraction process for polyphenol recovery using a natural deep eutectic solvent," Antioxidants, vol. 8, no. 12, p. 586, 2019.

[33] O. Kaltsa, S. Yanniotis, M. Polissiou, and I. Mandala, "Stability, physical properties and acceptance of salad dressings containing saffron (Crocus sativus) or pomegranate juice powder as affected by high shear (HS) and ultrasonication 
(US) process," LWT(Lebensmittel-Wissenschaft \& Technologie), vol. 97, pp. 404-413, 2018.

[34] S.-L. Huang and Y.-S. Lin, "The size stability of alginate beads by different ionic crosslinkers," Advances in Materials Science and Engineering, vol. 2017, Article ID 9304592, 7 pages, 2017.

[35] P. Smrdel, M. Bogataj, and A. Mrhar, "The influence of selected parameters on the size and shape of alginate beads prepared by ionotropic gelation," Scientia Pharmaceutica, vol. 76, no. 1, pp. 77-90, 2008.

[36] S. R. Kannat, S. Tari, and S. P. Chawla, "Encapsulation of extract prepared from irradiated onion scales in alginate beads: a potential functional food ingredient," Food Measure, vol. 12, pp. 848-858, 2018.

[37] A. Belscak -Cvitanoviv, D. Komes, S. Karlović et al., "Improving the controlled delivery formulations of caffeine in alginate hydrogel beads combined with pectin, carrageenan, chitosan and psyllium," Food Chemistry, vol. 167, pp. 378-386, 2015.

[38] C. D. Di Mattia, D. Sacchetti, D. Mastrcola, D. K. Sarker, and P. Pittia, "Surface properties of phenolic compounds and their influence on the dispersion degree and oxidative stability of olive oil O/W emulsions," Food Hydrocolloids, vol. 24, no. 6-7, pp. 652-658, 2010.

[39] B.-B. Lee, P. Ravindra, and E.-S. Chan, "Size and shape of calcium alginate beads produced by extrusion dripping," Chemical Engineering \& Technology, vol. 36, no. 10, pp. 1627-1642, 2013.

[40] W. D. Williams, "Origin and impact of color on consumer preference for food1," Poultry Science, vol. 71, no. 4, pp. 744-746, 1992.

[41] L. L. Garber, E. M. Hyatt, and R. G. Starr, "The effects of food color on perceived flavor," Journal of Marketing Theory and Practice, vol. 8, no. 4, pp. 59-72, 2000.

[42] J. Popov-Raljić and J. Lalicic, "Dietary chocolate colors during their storage up to 1 year," Journal of Agricultural Sciences, vol. 52, 2007.

[43] S. Aroyeun and G. Adegoke, "Potential of Aframomum danielli spice powder in reducing ochratoxin A in cocoa powder," American Journal of Food and Nutrition, vol. 1, pp. 155-165, 2011.

[44] C. Quiroz Reyes and V. Fogliano, "Design cocoa processing towards healthy cocoa products: the role of phenolics and melanoidins," Journal of Functional Foods, vol. 45, pp. 480-490, 2018.

[45] R. Stojanovic, A. Belscak-Cvitanovic, V. Manojlovic, D. Komes, V. Nedovic, and B. Bugarski, "Encapsulation of thyme (Thymus serpyllum L.) aqueous extract in calcium alginate beads," Journal of the Science of Food and Agriculture, vol. 92, no. 3, pp. 685-696, 2012.

[46] V. Jost, K. Kobsik, M. Schmid, and K. Noller, "Influence of plasticiser on the barrier, mechanical and grease resistance properties of alginate cast films," Carbohydrate Polymers, vol. 110, pp. 309-319, 2014.

[47] M. Avella, E. D. Pace, B. Immirizi, G. Impallomeni, M. Malinconico, and G. Santagata, "Addition of glycerol plasticizer to seaweeds derived alginates: influence of microstructure on chemical-physical properties," Carbohydrate Polymers, vol. 69, no. 3, pp. 503-511, 2007.

[48] A. Marismandani and A. Husni, "Development and characterization of biobased alginate/glycerol/virgin coconut oil as biodegradable packaging," E3S Web of Conferences, vol. 147, p. 03016, 2020.

[49] R.-M. Ion, "Evaluation of natural polyphenols entrapped in calcium alginate beads prepared by the ionotropic gelation method," Journal of Optoelectronics and Advanced Materials, vol. 15 , no. 7-8, pp. 893-898, 2013. 